\title{
Cost of treatment and reduction achieved by chronic ulcer in diabetic patients - a comparison between hydrogel and human recombinant Epidermal Growth Factor
}

\section{Gabriela Deutsch ( $\nabla$ gd85@globo.com )}

Universidade Federal Fluminense https://orcid.org/0000-0002-4618-439X

Bianca Campos de Oliveira

Universidade Federal Fluminense

Fernanda Soares Pessanha

Universidade Federal Fluminense

Keila Mara Cassiano

Universidade Federal Fluminense

Beatriz Guitton Renaud Baptista de Oliveira

Universidade Federal Fluminense

Selma Rodrigues de Castilho

Universidade Federal Fluminense

Research article

Keywords: Diabetic foot, Epidermal growth factor, Wound healing, cost effective

Posted Date: December 17th, 2019

DOI: https://doi.org/10.21203/rs.2.18921/v1

License: (c) (i) This work is licensed under a Creative Commons Attribution 4.0 International License.

Read Full License 


\section{Abstract}

Background: The study provides results from a double-blind clinical trial of 25 patients with diabetic or venous chronic ulcers. The main focus was to evaluate the assessing cost of outpatient treatment with two technologies: $2 \%$ carboxymethylcellulose hydrogel or human recombinant Epidermal Growth Factor carbogel.

Methods: Patients were randomly allocated to two ulcer treatment groups, human recombinant Epidermal Growth Factor carbogel treated (intervention group, $n=14$ ), and $2 \%$ carboxymethylcellulose hydrogel $(\mathrm{CMC})$ (control group, $\mathrm{n}=11$ ); both treated during 90 days. To calculate the costs of each patient's procedures, direct costs and human resources costs were measured in Brazilian currency and converted into US dollars. Statistical analysis of clinical data was performed based on intention to treat. For each treatment were analyzed the proportion of cases with wound reduction; cost benefit of the treatment given by the average value of each $1 \mathrm{~cm} 2$ wound reduction; statistics on the cost of reducing each $\mathrm{cm} 2$ of wound for each patient presenting wound reduction.

Results: In all evaluated periods, the proportion of cases with decreased lesion area was higher in the intervention group; It presented better cost-benefit ratio and lower average and median reduction cost for each reduced $\mathrm{cm} 2$ of the wound.

Conclusion: Data suggested that growth factor therapy may be cost-effective and innovative complement to standard wound care.

\section{Background}

Among the various chronic complications associated with diabetes mellitus, various conditions, such as those resulting from peripheral neuropathy and venous disease, can lead to chronic ulcers [1]. These lesions may progress with infection, osteomyelitis and amputation associated with significant morbidity, mortality and high costs $[2,3]$.

Currently, in addition to the comorbidities associated with diabetes, the increasing life expectancy of these patients also increases the incidence of these conditions [4]. In developing countries such as Brazil, evidence suggests that lifetime occurrence of diabetic ulcers is between $19 \%$ and $34 \%$ [5]. These injuries and amputation are more common in low- and middle-income countries [6].

The health costs of treating chronic ulcers in diabetic patients with venous leg ulcers and diabetic foot ulcers are five times higher than in diabetic individuals with no ulcer $[4,7]$. Those costs are mainly related to hospitalizations, more frequent in diabetics, but also to the care costs of treatment and follow-up of outpatient clinical management [8-11].

These injuries have received more and more attention from the multidisciplinary health team with the intention of developing advanced technology coverage in order to improve the healing result in the 
shortest possible time making it economically interesting [12]. These therapies costs and the scar deficiencies prevalence increase the importance of studies in search of new drugs and dressings capable of interacting with the injured tissue, aiming to accelerate the healing process $[13,14]$.

The topical Recombinant Human Epidermal Growth Factor (rhEGF) use has been highlighted in the wound healing process. It has been considered able to accelerate the rate of epidermal regeneration and reepithelialization with positive results in Asia and Central and South America, expanding its worldwide acceptance to Europe [15-21]. RhEGF stimulates the proliferation and migration of keratinocytes and fibroblasts, improving wound healing [22].

Because it is a protein, it has low stability and spontaneously degrades [23] and therefore several technologies have been tested to allow its topical application on the lesions. The use of advanced and innovative technologies can increase the cost of products.

In this sense, we sought to evaluate the cost of outpatient treatment of diabetic or venous ulcers of diabetic patients, using two technologies: $2 \%$ carboxymethylcellulose hydrogel (CMC) or rhEGF carbogel.

\section{Materials And Methods}

\subsection{Data base}

The database used for this analysis was originated in a randomized, double-blind, pragmatic clinical trial conducted at a public hospital located in Niteroi, Rio de Janeiro, Brazil. After approval by the Research Ethics Committee, all patients gave written informed consent and the collection process lasted 17 months starting in November 2016 and ending in April 2018. Participants were recruited for convenience during nursing consultations with diabetic outpatients who treated diabetic or venous chronic ulcers at the Wound Repair Outpatient Clinic of the hospital where the study was conducted. Follow-up time for each patient was 90 days. In addition to epidemiological data, detailed data on the material, supplies and covers used by each patient were retrieved from the clinical trial.

\subsection{Clinical Trial Development}

The study compared the cost of healing of diabetic and venous ulcers in diabetic patients using two different coatings: rhEGF carbogel or $2 \%$ carboxymethylcellulose hydrogel (CMC). Both products favor the healing of chronic ulcers. The $2 \% \mathrm{CMC}$ hydrogel is amorphous and used as a gold standard in the study hospital for the treatment of these lesions.

Randomization was performed by randomly allocating participants into the groups, Intervention (rhEGF carbogel) named product A and Control $(2 \% \mathrm{CMC})$ named product $\mathrm{B}$.

The patients were instructed on how to change the dressing daily, reapplying the product in sufficient quantity to cover the lesion with a thin layer. The weekly change in the outpatient clinic, as well as 
treatment of the injury was performed by the nurse. With this conduct, we sought the closest possible approximation to the reality of treatment of the patient.

The rhEGf carbogel needs to be stored in pump, airless vial and refrigerated for up to 60 days to maintain active stability. In order to blind the patients, both products were stored the same way and kept refrigerated.

Data related to product effectiveness and cost were collected. In all other nursing consultations, the wound was reevaluated, with the registration of the injury-specific data at weeks 01,06 and 12 . The participant and the two evaluators were blinded to the rate of reduction of the lesion area.

\subsection{Wound Reduction Rate}

The wound reduction rate (reduction / enlargement) analysis was calculated through formula 1, considering the area of the initial wound and the area after 12 weeks of treatment $[24,25]$.

[Due to technical limitations, the formula could not be displayed here. Please see the supplementary files section to access the formulas.]

To evaluate the reduction of the lesion area in square centimeters over the 12 weeks of treatment, the technique of manual planimetry and photography was performed. It consists of tracing the contour of the wound in sterile transparent material, calculating its final area by quantifying centimeter squares on squared paper [26].

\subsection{The economic assessment}

This study was based on the cost of a public agency in Brazil from the perspective of the Unified Health System (SUS). To calculate the treatment costs, data registration forms were established to observe how much material was used throughout the therapy considering both the outpatient care expenses and the material dispensed for home. The micro-costing survey was performed with all material dispensed for the patients' dressings. The calculation of these items was made from the average spent during treatment.

The home kits supplies were delivered according to the demand at each outpatient visit for dressing. The home dressing supplies observed were sterile hydrophilic gauze pad, crepe bandage, waterproof tape roll, vial containing $0.9 \%$ saline solution and needle $(40 \times 12)$. To perform the outpatient dressing, the same household inputs were observed plus disposable surgical mask, surgical cap, disposable procedure gloves, sterile surgical gloves, number 20 scalpel blade. This material was accounted for both during outpatient consultation and throughout weekly home use of the patient.

A survey was conducted to estimate the cost of supplies used by each patient in dressings by consulting purchase prices from a study hospital. An analysis includes direct costs: procedural costs considering test products, curative execution inputs, and human resource costs in outpatient consultations where the nursing time used in the consultation was recorded and calculated. As inputs, we considered the 
materials used in the dressing, at the outpatient clinic and at home. The measure used to account for or use was a Brazilian currency (Real). After accounting, the costs were converted to US dollars. For this conversion, a reference value was considered as an average between the highest closing value of the US dollar and the lowest locking value in May 2017, the month in which the cost data were collected in reais. According to ADVFN Brazil (Brazilian Stock Exchange Portal, with Bovespa and BM\&F quotes), or the highest closing value of the US dollar this month was recorded on May 18 (R\$ 3,33890), thirteenth trading session of the month and lowest closing value occurred on May 16 (R\$3.0955), eleventh trading session of the period. Therefore, the reference value used in the conversion was 1 US dollar for 3,24225 reais.

To evaluate the amount of gel used by the patients, a measure was made for home use, where the weekly return of the dispensed bottle was requested, which was weighed at the time of consultation, and its weight compared to the previous week. Outpatient use was measured at the time of dispensation.

During the weekly nursing consultation to perform dressings at the outpatient clinic, the time taken to perform this procedure was timed in minutes. The nursing workforce was calculated as the average between the base values of the fee table determined by Resolution 301/2005 of the Federal Nursing Council (COFEN), which regulates the profession, and the average value of the professional in the study hospital. To minimize the interpretation variables in the performance of the timing, it was previously determined the moment considered the beginning and end of the procedure, defined as the opening and closing of the patient's bandage at the outpatient clinic.

Statistical analysis of clinical data was performed based on the intention to treat. The choice of comparison tests between the groups was performed respecting the assumptions determined by the results, characteristics and behavior of the clinical study variables.

[See supp. files]

This calculation took into consideration the entire sample, including the cost of cases in which there was no reduction or one with increased lesion size. In the latter case, the value was entered as a negative reduction in the denominator, which decreases the denominator, penalizing the cost-benefit ratio as it should be.

Calculation of average (and other statistics) of cost of reduction of each $1 \mathrm{~cm}^{2}$ of wound

For each patient who presented injury reduction, the total cost of treatment for each patient in the period was calculated. This calculation was made through the cost of reducing each $1 \mathrm{~cm}^{2}$ in the period, divided by the total amount of reduced square centimeters in the same period.

Then, for each patient who presented wound reduction, reduction cost values of each $1 \mathrm{~cm}^{2}$ wound during the period were obtained and the statistics for this variable (minimum, maximum, average, median, standard deviation and coefficient of variation) were obtained. number of patients who presented wound reduction. 
In this calculation, the entire sample was not taken into account, since cases that had no reduction or when it was negative could not be included.

The coefficient of variation is calculated by the standard deviation divided by the mean.

The proportion of cases that presented wound reduction in each period with both treatments was analyzed. The distribution of the variable "cost of reduction of each $1 \mathrm{~cm}^{2}$ of the wound" was also calculated for each patient who presented injury reduction.

\section{Results}

Table 1 shows the frequency distribution of wound area variation over the evaluated time periods for both groups.

Table 1: Frequency distribution of the variation of the wound area in the evaluated time periods, for both groups, $\mathrm{N}=25$, Niteroi, 2018

\begin{tabular}{ccccc}
\hline Group & Wound Area Variation & First 6 weeks & Last 6 weeks & 12 weeks \\
\hline Control* & Increased the area & $1(9,1 \%)$ & $4(36,4 \%)$ & $4(36,4 \%)$ \\
& Did not change area & $1(9,1 \%)$ & $2(18,2 \%)$ & $1(9,1 \%)$ \\
& Decreased area & $\mathbf{9 ( 8 1 , 8 \% )}$ & $\mathbf{5 ( 4 5 , 5 \% )}$ & $\mathbf{6 ( 5 4 , 5 \% )}$ \\
Intervention** & Increased the area & $2(14,3 \%)$ & $3(21,4 \%)$ & $2(14,3 \%)$ \\
Did not change area & $0(0,0 \%)$ & $1(7,1 \%)$ & $0(0,0 \%)$ & \\
& Decreased area & $12(\mathbf{8 5 , 7 \% )}$ & $\mathbf{1 0 ( 7 1 , 4 \% )}$ & $\mathbf{1 2 ( 8 5 , 7 \% )}$ \\
\hline
\end{tabular}

* $2 \%$ carboxymethylcellulose hydrogel (CMC)

** rhEGF carbogel

In all periods considered, and for both control and intervention groups, there was at least 1 case of treatment failure, it means that, neither treatment was $100 \%$ effective in reducing the wound area over the 12 weeks. It was also possible to observe at least 1 case in which there was no alteration of the lesion area or in the same one that increased its area. 
Also according to table 1, the treatment failure for each period were estimated for both groups. The probability of no wound reduction in the first 6 weeks for the control group was 18.2\%; while in the intervention group it was 14.3\%. The same calculation was made for the later period of the last 6 weeks, where the control group resulted in $54.5 \%$ while in the intervention group it was $28.5 \%$.

The probability of no wound reduction in the 12 -week total study period was $45.5 \%$ in the control group and $14.3 \%$ for the intervention group.

Thus, in all evaluated periods, the probability of failure is higher for the control treatment. The results suggest that in all evaluated periods the probability of success is higher than the treatment intervention.

3.1 Calculation of cost-effectiveness of treatment given by the average cost of each $1 \mathrm{~cm}^{2}$ wound reduction.

Table 2 shows total reduction and average cost of each $1 \mathrm{~cm}^{2}$ wound reduction in the control and treatment groups in each period evaluated.

Table 2: Total cost, total reduction and average cost of each $1 \mathrm{~cm}^{2}$ wound reduction in the control and treatment groups in each period evaluated.

Variable

Sum of patient costs in this period (in U\$)

Sum of Reduced Areas over this period (in $\mathrm{cm}^{2}$ )

Average cost of $1 \mathrm{~cm}^{2}$ reduction over the period

\section{Period}

\begin{tabular}{|c|c|c|c|c|c|}
\hline \multicolumn{2}{|c|}{ First 6 weeks } & \multicolumn{2}{|c|}{ Last 6 weeks } & \multicolumn{2}{|c|}{ Total (12 weeks) } \\
\hline Control & Treatment & Control & Treatment & Control & Treatment \\
\hline
\end{tabular}

$1.877,50$

$2.066,56$

$1.335,06$

$1.727,08$

$3.212,57$

$3.793,65$

27,1

83,4

$-17,4^{*}$

25,0

9,7

108,4

\section{9,28}

24,78

$-$

69,08

331,19

35,00 
* In this group, the sum of the reduced areas in the last 6 weeks was negative, showing that, in fact, in total, the total area of wounds increased by $17.4 \mathrm{~cm}^{2}$ for the control group patients during this period.

It was observed that in the first 6 weeks, the control group spent US1877,50 dollars to obtain a total reduction of $27.1 \mathrm{~cm}^{2}$; In the same period, in the intervention group, an amount of U\$2066,56 was spent resulting in a total reduction of $83.4 \mathrm{~cm}^{2}$.

The average cost in this same period was $\mathrm{U} \$ 69,28$ per $1 \mathrm{~cm}^{2}$ reduced in the control group while in the intervention group the value was $\mathrm{U} \$ 24,78$ per $\mathrm{cm}^{2}$. These values suggest that the cost-benefit ratio in the first 6 weeks is more positive in the intervention group than in the control group.

The same calculations were performed for the last 6 weeks. During this period, U\$1727,08 was spent to achieve a total reduction of $25.0 \mathrm{~cm}^{2}$ in the intervention group, leaving an average cost of $\mathrm{U} \$ 69,08$ per $\mathrm{cm}^{2}$. These results suggest that it is more expensive to reduce $1 \mathrm{~cm}^{2}$ in the last 6 weeks.

For the control group such a relationship cannot be calculated, considering the total changes in the areas, wounds generally increased rather than reduced. The sum of the changes in area left an increase of $17.4 \mathrm{~cm}^{2}$.

Considering the total study period, over the 12 weeks, the total cost with the patients in the control group was U\$3212,57 dollars, for a total reduction of only $9.7 \mathrm{~cm}^{2}$.

In the same period, in the intervention group, a greater amount of U\$3793,65 was spent, however, it was possible to notice a total reduction of $108,4 \mathrm{~cm}^{2}$.

The average cost in the first 12 weeks was $U \$ 331,19$ per $\mathrm{cm}^{2}$ reduced in the control group and U\$35,00 per $\mathrm{cm}^{2}$ in the intervention group. These results suggest that the cost-benefit ratio at 12 weeks is better in treatment than in the control group.

3.2 Calculation of average (and other statistics) of wound reduction cost $1 \mathrm{~cm}^{2}$ 
As shown in table 3, considering only the cases that had wound reduction, according to the statistics displayed, it was possible to evaluate that in all periods, the proportion of cases that decrease the wound is higher in the intervention group. These results suggest that the likelihood of success, ie wound reduction, is greater with intervention than with control.

Table 3: Key statistics of the cost of each reduced $\mathrm{cm}^{2}$ in the wound in cases where there was a reduction in control and treatment groups in each period evaluated.

\begin{tabular}{|c|c|c|c|c|c|c|c|c|c|}
\hline \multirow[t]{2}{*}{ Period } & \multirow[t]{2}{*}{ Group } & \multirow[t]{2}{*}{$n^{*}$} & \multirow[t]{2}{*}{$p^{*}$} & \multicolumn{6}{|c|}{ Reduction cost statistics for each reduced wound $\mathrm{cm}^{2}\left(\mathrm{U} \$ / \mathrm{cm}^{2}\right)$} \\
\hline & & & & Minimum & Maximum & Mean & Median & Standard & C.V \\
\hline & & & & & & & & Deviation & \\
\hline \multirow[t]{2}{*}{ First 6 weeks } & Control & 9 & $81,8 \%$ & 13,42 & 440,98 & 132,87 & 66,11 & 139,89 & 1,05 \\
\hline & Intervention & 12 & $85,7 \%$ & 7,78 & 331,44 & 76,57 & 19,55 & 101,70 & 1,33 \\
\hline \multirow[t]{2}{*}{ Last 6 weeks } & Control & 5 & $45,5 \%$ & 42,33 & 333,29 & 180,35 & 178,51 & 110,34 & 0,61 \\
\hline & Intervention & 10 & $71,4 \%$ & 9,69 & 540,29 & 175,46 & 120,76 & 176,34 & 1,00 \\
\hline Total (12 & Control & 6 & $54,5 \%$ & 13,42 & 203,61 & 112,72 & 95,74 & 65,70 & 0,58 \\
\hline weeks) & Intervention & 12 & $85,7 \%$ & 9,02 & 534,42 & 110,96 & 38,26 & 150,75 & 1,36 \\
\hline
\end{tabular}

$n^{*}=$ number of patients who presented wound reduction in this period

$p^{*}=$ proportion of patients who had wound reduction in this period, estimation of the probability of a patient receiving treatment to have wound reduction in this period

In all periods evaluated, the mean reduction cost for each reduced wound $\mathrm{cm}^{2}$ is also lower in the intervention group. The same was observed for the median reduction cost for each reduced $\mathrm{cm}^{2}$.

The reduction cost of $1 \mathrm{~cm}^{2}$ presented high variability between patients in both groups in all evaluated periods, given the coefficients of variation well above 0.4 . 
Considering the total period of 12 weeks, it was observed that the chance of a patient undergoing the

intervention to have wound reduction is $85.7 \%$. However, this $1 \mathrm{~cm}^{2}$ reduction cost measure shows high variability $(\mathrm{CV}=1.36)$ in the range of 9,02 to $U \$ 534,42 / \mathrm{cm}^{2}$, with an average of $U \$ 110,96 / \mathrm{cm}^{2}$ and a median of $38,26 \mathrm{U} \$ / \mathrm{cm}^{2}$.

These results suggest that the chance of a patient undergoing control treatment to have wound reduction within 12 weeks of treatment is considerably lower, at only $54.5 \%$. The cost of reduction of $1 \mathrm{~cm}^{2}$ in this period presented high variability $(\mathrm{CV}=0,58)$ in the range of 13,42 to $203,61 \mathrm{U} \$ / \mathrm{cm}^{2}$, with an average of $\mathrm{U} \$ 112,72$ / median of $95,74 \mathrm{U} \$ / \mathrm{cm}^{2}$.

In all evaluated periods, the average and median reduction cost for each reduced wound $\mathrm{cm}^{2}$ is lower in the intervention group.

\section{Discussion}

Investigating the cost-benefit ratio of the treatments through the reduced average cost per $\mathrm{R} \$ \mathrm{sm}$ showed that the cost-benefit ratio in the first 06 weeks had better results in the intervention group, with $24078 \mathrm{U} \$ / \mathrm{cm}^{2}$ lower than in the control group with $69,28 \mathrm{U \$} / \mathrm{cm}^{2}$ reduced. Similar results were observed in the last 06 weeks as well, with favorable relation to the intervention group, since in the total sum there was no reduction in the control group.

Overall, the results suggest that it is more expensive to reduce $1 \mathrm{~cm}^{2}$ in the last 06 weeks of treatment than in the first 06 weeks. These findings are consistent with another study that suggests that early initiation of growth factor treatment in diabetic patients may lead to lower overall health costs, improving wound healing outcomes [27].

When observing the total period evaluated, referring to the 12 weeks of the study, it is more evident that the cost-benefit ratio is better in the intervention group with $35,00 \mathrm{U} \$ / \mathrm{cm}^{2}$ reduced than in the control group with 331,19 U\$ / $\mathrm{cm}^{2}$ reduced. This result is in agreement with the literature, since other studies show that rhEGF favors the increase of granulation tissue in the lesion bed, favoring the healing process $[19,28-31]$.

These results also corroborate the fact that treatments that accelerate the healing of chronic ulcers in diabetic patients reduce the need for hospitalization, and consequently the costs of care [27].

\section{Calculation of average (and other statistics) of wound reduction cost $1 \mathrm{~cm}^{2}$}


In all periods evaluated, the proportion of cases with decreased lesion area was higher in the intervention group. These findings suggest that the likelihood of success, ie wound reduction, is greater when treated with rhEGF (intervention product) than with control. Other studies using rhEGF in the intervention also observed similar results [18, 32].

\section{Conclusions}

Chronic ulcers in diabetic patients have a serious impact on the global burden of disease. The treatment of these patients generates high costs due to factors such as: possibility of long hospital stay, incident need for rehabilitation, frequently needed home care and the use of social services and possible complications of diabetic foot such as amputation.

Although the short follow-up period and the small number of patients, the main limitations of this study, require caution in extrapolating these results, they suggest that growth factor therapy may be a costeffective and innovative complement to standard wound care.

\section{List Of Abbreviations}

Carboxymethylcellulose hydrogel (CMC)

National Health Council (CSN)

Recombinant Human Epidermal Growth Factor (rhEGF)

Unified Health System (SUS)

ADVFN Brazil (Brazilian Stock Exchange Portal, with Bovespa and BM\&F quotes)

Federal Nursing Council (COFEN)

\section{Declarations}

Funding: The authors thank Faperj and CNPq for granting doctoral and scientific initiation scholarships.

Acknowledgments: The authors thank Infinity pharma for the assignment of rhEGF and the hospital material.

Conflicts of Interest: The authors declare no conflict of interest. The funders had no role in the design of the study; in the collection, analyses, or interpretation of data; in the writing of the manuscript, or in the decision to publish the results.

Ethical responsibility: These research was submitted to the Ethics Committee of the Fluminense Federal University Medical School, respecting the evaluation methods in Resolution N $N^{0} .466 / 12$ of the National Health Council (CSN) of the Ministry of Health and approved ( $N^{\circ} .1,602,173$ on 06/16/2016). 
Author Contributions: G.D, B.C.O, B.G.R.B.O, and S.R.C worked at conceptualization; G.D, B.C.O, B.G.R.B.O, and S.R.C contributed to the methodology; The investigation was made by G.D, B.C.O, and F.S.P; Data curation was cataloged by G.D, B.C.O, and F.S.P; The formal analysis were analyzed by K.M.C; writing the original draft preparation was made by G.D, B.C.O, and F.S.P; And the writing-review and editing was made by B.G.R.B.O, and S.R.C; Funding acquisition was made by G.D, B.G.R.B.O, and S.R.C.

\section{References}

1. Lucoveis, M.D.L.S., et al., Degree of risk for foot ulcer due to diabetes: nursing assessment. Revista Brasileira de Enfermagem, 2018. 71: p. 3041-3047.

2. Bakker, K., et al., The 2015 IWGDF guidance documents on prevention and management of foot problems in diabetes: development of an evidence-based global consensus. Diabetes Metab Res Rev, 2016. 32 Suppl 1: p. 2-6.

3. Gutknecht, M., et al., Costs Of Compression Therapy In Venous Leg Ulcers In Germany And Modelling Of The Economic Effects Of Regional Disparities In Health Care. Value Health, 2015. 18(7): p. A372.

4. Toscano, C.M., et al., Annual Direct Medical Costs of Diabetic Foot Disease in Brazil: A Cost of IIIness Study. Int J Environ Res Public Health, 2018. 15(1).

5. Armstrong, D.G., Boulton, A.J.M, and S.A. Bus, Diabetic Foot Ulcers and Their Recurrence. N Engl J Med, 2017. 376(24): p. 2367-2375.

6. Nussbaum, S.R., et al., An Economic Evaluation of the Impact, Cost, and Medicare Policy Implications of Chronic Nonhealing Wounds. Value Health, 2018. 21(1): p. 27-32.

7. Motley, T.A., et al., Cost-effectiveness of clostridial collagenase ointment on wound closure in patients with diabetic foot ulcers: economic analysis of results from a multicenter, randomized, openlabel trial. J Foot Ankle Res, 2015. 8: p. 7.

8. Donnan, P.T., Leese, G.P, and A.D. Morris, Hospitalizations for people with type 1 and type 2 diabetes compared with the nondiabetic population of Tayside, Scotland: a retrospective cohort study of resource use. Diabetes Care, 2000. 23(12): p. 1774-9.

9. Lugo-Palacios, D.G. and Cairns, J. The financial and health burden of diabetic ambulatory care sensitive hospitalisations in Mexico. Salud Publica Mex, 2016. 58(1): p. 33-40.

10. Hong, J.S. and Kang, H.C Continuity of ambulatory care and health outcomes in adult patients with type 2 diabetes in Korea. Health Policy, 2013. 109(2): p. 158-65.

11. Cavassini, A.C., et al., Cost-benefit of hospitalization compared with outpatient care for pregnant women with pregestational and gestational diabetes or with mild hyperglycemia, in Brazil. Sao Paulo Med J, 2012. 130(1): p. 17-26.

12. Lima, R.V.K.S., Coltro, P.S and Farina Júnior, J.A. Negative pressure therapy for the treatment of complex wounds. Revista do Colégio Brasileiro de Cirurgiões, 2017. 44: p. 81-93.

13. Mendonça, R.J.d. and J. Coutinho-Netto, Aspectos celulares da cicatrização. Anais Brasileiros de Dermatologia, 2009. 84: p. 257-262. 
14. Lindholm, C. and Searle, R. Wound management for the 21st century: combining effectiveness and efficiency. International Wound Journal, 2016. 13(S2): p. 5-15.

15. Garcia-Orue, I., et al., Novel Nanofibrous Dressings Containing Rhegf And Aloe Vera For Wound Healing Applications. Int J Pharm,2017; 523(2):556-566.

16. Kim, H., et al., Hyaluronate-Epidermal Growth Factor Conjugate for Skin Wound Healing and Regeneration. Biomacromolecules, 2016; 17(11):3694-3705.

17. Goh, M., Hwang, Y. and Tae, G. Epidermal growth factor loaded heparin-based hydrogel sheet for skin wound healing. Carbohydrate Polymers, 2016. 147: p. 251-260.

18. Park, K.H., et al., Topical epidermal growth factor spray for the treatment of chronic diabetic foot ulcers: A phase III multicenter, double-blind, randomized, placebo-controlled trial. Diabetes Res Clin Pract, 2018. 142: p. 335-344.

19. Esquirol-Caussa, J. and Herrero-Vila, E. Human recombinant epidermal growth factor in skin lesions: 77 cases in EPItelizando project. J Dermatolog Treat, 2018: p. 1-6.

20. Xu, J., et al., Experimental study of epidermal growth factor and acidic fibroblast growth factor in the treatment of diabetic foot wounds. Exp Ther Med, 2018. 15(6): p. 5365-5370.

21. Ertugrul, B.M., Lipsky,B.A. and Guvenc, U. An Assessment of Intralesional Epidermal Growth Factor for Treating Diabetic Foot WoundsThe First Experiences in Turkey. J Am Podiatr Med Assoc, 2017. 107(1): p. 17-29.

22. Bodnar, R.J., Epidermal Growth Factor and Epidermal Growth Factor Receptor: The Yin and Yang in the Treatment of Cutaneous Wounds and Cancer. 2013(2162-1918 (Print)).

23. Araki, F., et al., Stability of recombinant human epidermal growth factor in various solutions. Chem Pharm Bull (Tokyo), 1989. 37(2): p. 404-6.

24. Taradaj, J., et al., Evaluation of efficiency of compression therapy in enhancement of venous leg ulcer healing in patients after surgical treatment. 2008, EWMA Journal Electronic Supplement.

25. Guitton, B.R.O.B., De Abreu, C.J.B. and Carvalho, A.N Técnicas Utilizadas na Aferição de Feridas e Avaliação do Processo CicatricialSaúde Coletiva. 2005, Saúde coletiva. p. 57-62.

26. Silveira, I.A., Leg Ulcer Measurement Tool: tradução e adaptação transcultural para a língua portuguesa. 2016.

27. Lantis, J.C., 2nd, et al., Analysis of patient cost for recombinant human platelet-derived growth factor therapy as the first-line treatment of the insured patient with a diabetic foot ulcer. Adv Skin Wound Care, 2009. 22(4): p. 167-71.

28. Yang, S., et al., Efficacy of Topical Recombinant Human Epidermal Growth Factor for Treatment of Diabetic Foot Ulcer: A Systematic Review and Meta-Analysis. Int J Low Extrem Wounds, 2016. 15(2): p. $120-5$.

29. Garcia Herrera, A.L., et al., Curative Metatarsal Bone Surgery Combined with Intralesional Administration of Recombinant Human Epidermal Growth Factor in Diabetic Neuropathic Ulceration 
of the Forefoot: A Prospective, Open, Uncontrolled, Nonrandomized, Observational Study. Curr Ther Res Clin Exp, 2017. 85: p. 2-7.

30. Isikgoz Tasbakan, M., et al., Intralesional epidermal growth factor therapy fordiabetic foot ulcers: an evaluation of 15 cases. Turk J Med Sci, 2017. 47(5): p. 1500-1504.

31. Esquirol Caussa, J. and Herrero Vila, E. Epidermal growth factor, innovation and safety. Medicina Clínica (English Edition), 2015. 145(7): p. 305-312.

32. Fernandez-Montequin, J.I., et al., Intralesional administration of epidermal growth factor-based formulation (Heberprot-P) in chronic diabetic foot ulcer: treatment up to complete wound closure. 2009(1742-481X (Electronic)).

\section{Supplementary Files}

This is a list of supplementary files associated with this preprint. Click to download.

- Methodsformulas.docx

- DatabaseGabrielaDeutsch.xIsx 